\title{
¿Overscreening o prevención a escala humana? Tamizaje excesivo
}

\author{
Sobrerrastreamento ou prevenção em uma escala humana? Excesso de rastreamento
}

\section{Overscreening or prevention in a human scale? Excessive screening}

Miguel Pizzanelli Báez. Especialista en Medicina Familiar y Comunitaria. Coordinador de la Unidad docente asistencial Rural de Florida, Uruguay. Profesor Adjunto del Departamento de Medicina Familiar y Comunitaria de la Facultad de Medicina de la UDELAR. Florida, Uruguay. miguelpizzanelli@gmail.com (Autor correspondiente)

\section{Resumen}

Cuando se aplica un método de cribado o tamizaje de modo excesivo, abusivo o innecesario los daños provocados pueden superar a los beneficios. Para desarrollar el concepto de tamizaje excesivo, fueron consideradas algunas categorías: tamizaje innecesario, sin indicación médica, inducido, obligatorio, y por frecuencia inadecuada. Existen sesgos de interpretación que no permiten determinar de forma objetiva el balance daño beneficio de los programas de tamizaje relacionados al exceso de diagnóstico. Evitar el daño producido por tamizajes excesivos (overscreening) requiere llevar recomendaciones y pautas genéricas al terreno de la práctica clínica con casos particulares. La prevención cuaternaria, fundada conceptualmente en los cuidados de salud centrados en la persona, permite considerar las creencias, inquietudes, opciones individuales, haciendo posible llevar a la prevención a una escala humana.

\section{Resumo}

Quando um método de rastreio é aplicado de forma excessiva, desnecessária ou abusiva, os danos podem superar os benefícios. Para desenvolver 0 conceito de excesso de rastreamento, foram consideradas algumas categorias: rastreamento desnecessário, sem indicação médica, induzido, obrigatório, e por frequência inadequada. Existem vieses de interpretação que não permitem determinar objetivamente 0 balanço dos danos e benefícios dos programas de rastreamento relacionados ao excesso de diagnósticos. Para se evitar danos causados por excesso de rastreamento (overscreening) é necessário se deslocar do campo das recomendações e pautas genéricas para o terreno da prática clínica, com casos particulares. A prevenção quaternária, fundamentada conceitualmente nos cuidados de saúde centrados na pessoa, permite considerar as crenças, interesses, opções individuais, tornando possível levar a prevenção a uma escala humana.

\section{Abstract}

When screening is applied in an excessive, unnecessary or abusive manner, the damage may outweigh the benefits. In order do develop the concept of excessive screening some categories were considered: unnecessary screening, screening without medical indication, induced screening, mandatory screening, and inadequate frequency screening. There are biases of interpretation that do not allow objectively determining the balance of harms and benefits of screening programmes related to overdiagnosis. To prevent damage from excessive screening (overscreening) it is necessary to move from the domain of generic recommendations and guidelines to the clinical practice ground of individual cases. The quaternary prevention, conceptually grounded in person-centred health care, comprises the beliefs, interests, and individual options, making it possible to lead prevention to a human scale.
Palabras clave: Sobretamizaje Tamizaje Masivo Relaciones Médico-Paciente Prevención Cuaternaria Medicalización

\section{Palavras-chave:}

Sobrerrastreamento Programas de Rastreamento Relações Médico-Paciente Prevenção Quaternária Medicalização

\section{Keywords:} Overscreening Mass screening Physician-Patient Relations Quaternary Prevention Medicalization 


\section{¿Overscreening o prevención a escala humana? Tamizaje excesivo}

Los programas de cribado o tamizaje son actividades incluidas en el nivel secundario de prevención y se aplican de forma masiva y sistemática en población sana asintomática. Su objetivo es reducir la morbilidad y fundamentalmente la mortalidad de una enfermedad mediante la aplicación de algún tipo de intervención en etapa pre sintomática desde una perspectiva de disminución de riesgos. ${ }^{1}$ Es preciso distinguir entre los conceptos de tamizaje (o cribado) y diagnóstico o detección precoz. Tamizaje, como se ha dicho implica una intervención masiva, desde el sistema de salud hacia una población de riesgo. Por diagnóstico precoz entendiese una acción individualizada, centrada en la persona. Así el diagnóstico precoz pretende detectar un problema de salud en una etapa o fase inicial y ofrecer mejores chances de tratamiento, cura y sobrevida. Otra modalidad frecuente de tamizaje es llamado oportunista. Este ocurre cuando el profesional de salud aprovecha la consulta de un usuario por otro motivo, para plantearle la búsqueda de una condición o factor de riesgo. ${ }^{2}$

Las controversias éticas respecto a muchos programas de tamizaje o screening y a los excesos en los tamizajes, giran en torno a varias premisas fundamentales. En este artículo se discuten solamente tres de ellas. En primer lugar, un programa masivo de screening para ser justificable debe asegurar que la intervención propuesta brinde mayores beneficios que dańos. Al ser aplicado en personas sanas y siendo que la iniciativa surge desde el sistema de salud y no desde los usuarios, debe contarse con certezas respecto a las garantías de los beneficios que éste ofrece sobre los daños que puede provocar. ${ }^{3}$ El segundo aspecto está centrado en la equidad. Este argumento debe ser tenido en cuenta particularmente, cuando se analiza la situación en países de economías emergentes, dado que los programas poblacionales o los tamizajes oportunistas pueden implicar consumo y desviación de recursos, siempre finitos, interfiriendo en el desarrollo de políticas y o acciones sanitarias, en áreas prioritarias de la salud colectiva.

El último aspecto tiene que ver con el papel de los profesionales de la salud en la comunicación con los usuarios. Un resultado positivo en un tamizaje unicamente está clasificando a un individuo en un grupo de 'probable mayor riesgo'. Deben ser consideradas otras dimensiones como la sensibilidad y especificidad de la prueba, las consecuencias de resultados falsos positivos o negativos y los sesgos posibles cuando se comunican los resultados poblacionales de los programas de screening. ${ }^{3}$ La comunicación efectiva, el vínculo en el binomio médico/paciente, los cuidados centrados en la persona, la toma de decisiones compartidas, son herramientas claves en esta dimensión comunicacional para lograr compartir y dialogar sobre estos conceptos complejos de modo individualizado y asertivo. Este artículo presenta consideraciones sobre excesos en los tamizajes, para lo cual se comienza por definir overscreening.

\section{Definición}

Dado el amplio uso que se da al término screening en el lenguaje médico y de la salud, se analizó en su sentido semántico para intentar una definición aceptable. Este anglicismo es el gerundio del verbo to screen. Entre las traducciones posibles de este verbo, que aplican a nuestro interés particular, se encuentran las siguientes acepciones: ${ }^{4}$

- Tamizar, pasar por una criba; (suspects) etc investigar; (select) seleccionar, pasar por el tamiz. Acción de filtrar o tamizar.

- (Med) hacer una exploración a. (textual de diccionario).

Por lo tanto, 'to screen' refiere a un proceso que permite seleccionar e identificar individuos pertenecientes a una población, de acuerdo a algún criterio preestablecido.

La primera vez en ser utilizada la palabra overscreening, con el sentido de tamizaje excesivo, fue en 1989 en una publicación de Petr Scrabanek en la que se promovía el debate racional en el uso de mamografías como método de cribado para cáncer de mama. ${ }^{5}$ Posteriormente, en 1992, fue utilizado en una publicación sobre tamizaje de cáncer de cuello de útero indexada en PUBMED. ${ }^{6}$ De todos modos el término overscreening no dispone de descriptor MeSH en esta base de datos. $^{7}$ Se propone la siguiente definición para overscreening, con el sentido de cribado o tamizaje en exceso: 
Overscreening define aquellas situaciones en las que se emplea un método de cribado o tamizaje de un modo excesivo, abusivo o innecesario de tal forma que los daños que provoca superan a los beneficios.

\section{Ejemplos de tamizaje en exceso}

Debido al impacto sanitario del cáncer como enfermedad, a la intensidad con que se le busca y por ser motivo de preocupación de usuarios, pacientes y profesionales de la salud, en este trabajo se hace referencia, fundamentalmente al tamizaje en exceso en el caso de enfermedades oncológicas. Existen varias situaciones en las que se puede estar frente a lo que se denomina tamizaje en exceso.

\section{Tamizaje innecesario}

El hecho de categorizar a una práctica de tamizaje como innecesaria hace reflexionar sobre cuanta energía deben poner los profesionales de salud en actividades que no redundan en mejorar la salud percibida, y no se centran en el aumento de la calidad de vida y el disfrute vital de las personas. La palabra innecesario no expresa con claridad la capacidad de inducción de daño potencial que poseen estas prácticas. El nivel de daño puede inferirse por las consecuencias derivadas de los resultados posibles. Por ejemplo, el número de falsos positivos o la carga de sobrediagnóstico a la que se exponen las personas. A su vez estos fenómenos mencionados derivados del tamizaje a gran escala, tienen impacto en las decisiones que deben tomarse con cada individuo, así como en los costos tangibles e intangibles que provocan todas las intervenciones diagnósticas y terapéuticas que se desencadenan. Muchos de estos resultados no pueden ser evaluados en su verdadera magnitud, por tener características cualitativas de difícil ponderación. Por ejemplo: ¿qué impacto individual tiene recibir el rótulo de 'portadora o portador de cáncer'?8 A continuación se presentan algunos ejemplos de tamizaje innecesario.

Los exámenes para detección de cáncer en adultos mayores son innecesarios y provocan medicalización de la vida en exceso. ${ }^{9}$ Siendo el cáncer una causa frecuente de muerte en esta población, es evidente que cuanto más le busquemos, más le encontraremos. Otro ejemplo icónico de tamizaje innecesario, es el de la búsqueda del cáncer de próstata a través del antígeno prostático específico en sangre (PSA). Hay suficiente evidencia para no sostener este tamizaje por los daños que provoca y el escaso o nulo impacto en 'salvar vidas' que ofrece. De todos modos, muchas instituciones y grupos de profesionales siguen promoviendo su realización y no es tan simple argumentar en su contra, incluso para organizaciones consideradas poderosas. En 2009 y nuevamente en 2010 el US Preventive Services Task Force (USPSTF), concluyó que con moderada certeza el screening con PSA para cáncer de próstata no ofrecía beneficio neto, pero estas conclusiones nunca fueron publicadas. Finalmente en octubre de 2010, luego de que en un artículo del New York Times Magazine se dejara saber que las recomendaciones que se habían realizado casi dos años antes no se habían comunicado, una revisión realizada por un comité independiente de expertos de la institución apareció a la luz. ${ }^{10-12} \mathrm{La}$ recomendación actual de USPSTF en su guía del año 2012 es contraria a la realización de esta prueba con un nivel de recomendación tipo D. ${ }^{13}$

\section{Tamizaje sin indicación médica y tamizaje inducido}

Instituciones dedicadas a la venta de servicios tecnológicos o entidades aseguradoras, ofrecen chequeos para detección de enfermedades, pero también los ofrecen los proveedores de servicios de salud. Muchas personas acceden a este tipo de práctica alentadas por el supuesto de que 'más es mejor' o porque su estatus social lo impone como práctica saludable. Este tipo de 'productos' que proceden de una medicina altamente mercantilizada y que promueve el consumo, llevan a prácticas nocivas como las tomografías corporales totales con el riesgo que implican estos estudios al ser realizados sin indicación y en repetidas ocasiones. Actualmente se supera este riesgo con el acceso a tecnología que no utiliza radiación pero que expone a los usuarios a otros problemas como la detección de incidentalomas. Estos hallazgos fortuitos son muchas veces parte del llamado efecto reservorio. ${ }^{14}$ Esto implica la detección fortuita de 'anomalías' que no se asocian con molestias, alteraciones funcionales, enfermedad o muerte. 
Los medios masivos de comunicación juegan un rol destacado en la inducción de tamizajes en la población. La metodología utilizada incluye la promoción del miedo a enfermedades, la persuasión mediante generalizaciones que pueden ser verdades a medias o argumentos falaces. Se utilizan modelos de emulación de alto impacto mediático. Un ejemplo de esto último es conocido como 'Efecto Angelina'. ${ }^{15}$ La confiabilidad y calidad de las fuentes de información utilizadas por los medios de comunicación están frecuentemente sesgadas y afectadas por conflicto de intereses.

\section{Tamizaje obligatorio}

Algunos programas masivos son estimulados e instituidos desde el sistema de salud, por leyes o reglamentaciones. En otras ocasiones, son impuestos por usos y costumbres institucionales o a través de presiones de grupos de poder. Estas prácticas reglamentadas, al ser obligatorias no admiten proteger el principio ético de autonomía y autodeterminación de los individuos.

Hay una tensión dialéctica cuando se confronta entre el bien común y el ejercicio del derecho individual en las decisiones sobre el propio cuerpo y la vida. Es un aspecto que puede ser discutible, pero si un individuo no acepta realizar una práctica establecida, por ejemplo cumplir con un plan de vacunación y esa acción tiene efectos negativos en la comunidad, esto suele desencadenar sanciones o acciones compulsivas desde el sistema, tendientes a obligar el cumplimiento de la norma. Queda sin efecto esta discusión cuando la decisión afecta al individuo y su cuerpo sin provocar dańos a terceros, como suele ocurrir en condiciones crónicas no transmisibles. ${ }^{16}$

Cuando se debe cumplir con una práctica que está instituida como regla por el sistema de salud, cada profesional que brinda consejo a los usuarios, está en un punto de gran tensión ética, ya que debe enfrentar su propio conflicto interior entre lo que su conocimiento y experiencia le dicen y lo que está obligado a cumplir por mandato. Esto ocurre a los profesionales médicos cada vez que una trabajadora uruguaya solicita realizar su mamografía para cumplir con la reglamentación que impone el carné de salud. ${ }^{17}$

\section{Tamizaje en exceso por su frecuencia}

La probabilidad de tener un resultado falso positivo al realizar una prueba de cribado depende de factores relacionados con el tipo de prueba, su efectividad o calidad y características de la población a la que se aplica. Pero cuando se está ante un programa que implica cribados regulares con una secuencia temporal entre ellos, la probabilidad de obtener un resultado falso positivo es diferente a la que ocurre al analizar un resultado aislado. Esta probabilidad se hace acumulativa a lo largo del tiempo y depende del número de repeticiones. Con el número de repeticiones, el porcentaje esperado de falsos positivos aumenta de forma considerable. Es posible minimizar este efecto eligiendo una prueba con menor tasa de falsos positivos o ajustando la frecuencia con la que se sugiere realizar la prueba. Este es el caso del Papanicolaou (PAP). Si luego de dos o tres pruebas normales, estas se realizan a intervalos menores a 2 o 3 años ocurrirá mayor cantidad de falsos positivos. Por tanto se deben afrontar junto a las pacientes las consecuencias de este efecto estadístico, en la vida real. ${ }^{18}$

\section{¿Qué ocurre cuando se realizan tamizajes excesivos?}

\section{Sobrediagnóstico y efecto en la sobrevida a 5 años}

Lo que llamamos cáncer define en realidad un concepto patológico bastante complejo y heterogéneo. Incluye entidades que evolucionan a la letalidad, procesos indolentes que no causan daño durante la vida, e incluso lesiones que involucionan. ${ }^{19,20}$ Cuando esta enfermedad evoluciona rápidamente el tamizaje es inefectivo y cuando no se manifiesta por ser indolente, la detección es fuente de daño por sobrediagnóstico y tratamiento innecesario. ${ }^{21}$ Los tamizajes incorporan a la casuística, personas a las que se diagnostica cáncer en etapas tempranas, y esto aumenta el número de sobrevivientes a cinco años, lo que produce una sobreestimación en este indicador. No sabemos cuántas de estas personas realmente se habrían vuelto sintomáticas en la evolución de su enfermedad. Tampoco hay manera de saber quiénes han sido sobrediagnosticados. Aunque el tratamiento haya sido totalmente inefectivo y como este factor no puede ser analizado, la sobrevida a cinco años aumentará, lo que reforzara el argumento a favor de los programas de cribado. ${ }^{22}$ Este efecto ocurre con cribados para cáncer de mama, próstata, tiroides y piel en los que la incidencia ha aumentado sin que ocurran cambios significativos en la mortalidad. ${ }^{23,24}$ 


\section{Sesgo en el valor predictivo positivo de una prueba}

Otro sesgo en la interpretación de los beneficios que ofrecen los cribados, es el efecto que ocurre sobre la sensibilidad del estudio utilizado: el sobrediagnóstico aumenta la sensibilidad haciendo aumentar falsamente el valor predictivo positivo del mismo.

\section{Riesgo relativo versus riesgo absoluto}

Cuando se comunica información sobre los beneficios o efectos de los tamizajes masivos en determinada población, se suele hacer a través de datos sobre riesgo relativo en lugar de utilizar el riesgo absoluto. El siguiente ejemplo muestra con mayor claridad esta situación: para un hombre de 55 años la disminución del riesgo relativo al recibir cribado para cáncer de colon es de $18 \%$. La reducción del riesgo absoluto (probabilidad de sufrir un evento), sin embargo, es tan solo de 0,014\%. Al discutir sobre los riesgos y beneficios de este tamizaje con los pacientes se puede argumentar que la probabilidad de no morir de cáncer colorrectal es de 99,34\% en la población cribada y de 99,20 en la no cribada. Como se ve, al analizar valores absolutos, la diferencia es ínfima y con los valores relativos parece abultada. ${ }^{25,26}$

\section{Falsos positivos}

El análisis del efecto de los falsos positivos merecería un capítulo aparte. Existe mucha bibliografía sobre situaciones adversas derivadas de los mismos. Por ejemplo en el caso del cribado para cáncer de colon, mediante la detección de sangre oculta en heces, la sensibilidad y especificidad de esta prueba puede alterarse por el uso de ácido acetil salicílico. ${ }^{27}$

\section{¿Cómo avanzar? La escala humana de la prevención}

El exceso en los tamizajes depende de la planificación, promoción y aplicación de programas preventivos verticales, que responden a una modalidad de abordaje sanitario centrado en la enfermedad. Por medio de este enfoque reduccionista se pretende llegar a la salud colectiva a través de la gestión del riesgo individual. Así consideradas las actividades sanitarias, imponen de forma arrogante un saber por encima de otro. ${ }^{28}$ Esta forma de proceder en las relaciones de poder entre los actores en el sistema de salud es característica del modelo médico hegemónico tal cual lo describe Menéndez. ${ }^{29}$ El autor considera aspectos económicos, políticos, institucionales e ideológicos para conceptualizar y definir al modelo médico hegemónico del siguiente modo:

Las principales características estructurales del modelo médico son su biologismo, individualismo, ahistoricidad, a-sociabilidad, mercantilismo y eficacia pragmática, y si bien dichos rasgos pueden ser observados en la medicina practicada antes del siglo $X I X$, durante este siglo se profundizarán y potenciarán esos rasgos hasta convertirse en las caracteristicas dominantes de la biomedicina. Es importante subrayar que el biologismo articula el conjunto de los rasgos señalados y posibilita la exclusión de las condiciones sociales y económicas en la explicación de la causalidad y desarrollo de las enfermedades. El biologismo es el que posibilita proponer una historia natural de la enfermedad en la cual la historia social de los padecimientos queda excluida o convertida en variables bioecológicas. $(\mathrm{p} .12)^{29}$

Este paradigma imperante establece un marco en el que se desarrollan prácticas funcionales a un modelo de salud mercantilizado. Este modelo se rige por leyes de consumo y por tanto admite tensiones en múltiples sentidos que proceden del mercado, la industria, los prestadores de salud y de los 'consumidores'.

\section{El nivel relacional clave para evitar los tamizajes en exceso y los excesos de los tamizajes}

Intentar evitar 'overscreenig' (o sobretamizaje) y el daño que produce, requiere acciones complejas en múltiples niveles. Se focalizará en las acciones de la dimensión relacional del binomio médico-paciente/paciente-médico. Es el llamado nivel micro del enfoque de prevención cuaternaria. ${ }^{30}$ En este escenario interpersonal se juega definitivamente la aplicación de la ética en la práctica médica a escala humana. 
Evitar excesos en los tamizajes requiere llevar recomendaciones y pautas genéricas al terreno de la práctica con casos particulares. La función de los médicos familiares y comunitarios implica, hacerse cargo de una larga lista de tareas que se extiende más allá de la aplicación de programas masivos para cribado de enfermedades. La interacción asertiva es crucial y requiere del profesional la habilidad de intentar acceder al conocimiento integral de cada individuo en su contexto. Este esfuerzo le permitirá asomar a la peculiaridad y originalidad humana. Solamente de ese modo se producirá una aproximación respetuosa hacia el otro, reconociendo diferencias, aceptando la asimetría y entendiendo el valor inclusivo de la alteridad. Así los médicos familiares y comunitarios estarán habilitados a realizar responsablemente la sugerencia precisa. Esta desafiante e interactiva forma de proceder admite considerar las preferencias, favorece la retroalimentación, para acordar un plan individualizado a seguir.

La prevención cuaternaria, fundada conceptualmente en los cuidados de salud centrados en la persona, permite considerar las creencias, inquietudes, opciones particulares y propias de cada individuo, haciendo posible una dimensión humana en la prevención. Promueve que cada persona participe de acuerdo a su situación, sus características y dones, en la toma de decisiones vinculadas a su dimensión de salud, respetando los principios éticos y específicamente los principios de autonomía y no maleficencia (primun non nocere). Incorporar la mirada que promueve la prevención cuaternaria en la práctica médica permite: (1) reorientar el enfoque de prevención del eje cronológico hacia el relacional; (2) moverse desde un modelo preventivo centrado en la enfermedad hacia uno centrado en la persona; y (3) aplicar desde esta perspectiva una organización de prácticas en la que cada protagonista asume de forma corresponsable el lugar que ocupa.

Nota del autor: debido a las dificultades en encontrar términos aceptables para todos los idiomas implicados, se han utilizado en este trabajo como sinónimos: screening, cribado y "tamizaje" (que formalmente en castellano es un neologismo, pero que para el lenguaje médico se usa y se entiende bien).

\section{Agradecimientos}

A Marc Jamoulle por su perseverante actitud de desafiarme a escribir. Agradezco a Agustina Terra y Armando Norman por sus aportes y correcciones.

\section{Referencias}

1. Ministério da Saúde (BR), Secretaria de Atenção à Saúde. Departamento de Atenção Básica. Rastreamento. Brasília: Ministério da Saúde; 2010. (Série A. Normas e Manuais Técnicos Cadernos de Atenção Primária, n. 29).

2. Norman HA, Tesser CD. Rastreamento de doenças. In: Gusso GDF, Lopes JMC. Tratado de medicina de família e comunidade: princípios, formação e prática. Porto Alegre: Artmed; 2012. p. 521-532.

3. Gates TJ. Screening for cancer: evaluating the evidence. Am Fam Physician. [Internet] 2000 [última consulta el 2015 Jun 17] ;63(3):513-522. Disponible en: http://www.aafp.org/afp/2001/0201/p513.pdf.

4. Colin S. Collins Spanish-English, English-Spanih dictionary. 3a ed. Barcelona: Ediciones Grijalbo; 1994.

5. Skrabanek P. Shadows over screening mammography. Clin Radiol. 1989;40(1):4-5. http://dx.doi.org/10.1016/S0009-9260(89)80003-0. PMid:2920517.

6. McMaster H, Arroll B. Screening for cervical cancer: attitudes and policies among Auckland general practitioners. N Z Med J. 1992;105(931):125127. PMid:1560922.

7. MeSH Browser [Internet]. Bethesda (MD): National Library of Medicine (U.S.); 2002 [última consulta el 2014 Oct 25]; [1 pantalla]. Disponible en: http://www.ncbi.nlm.nih.gov/mesh/?term=overscreening.

8. Brodersen J, Siersma VD. Long-term psychosocial consequences of false-positive screening mammography. Ann Fam Med. 2013;11(2):106-115. http://dx.doi.org/10.1370/afm.1466. PMid:23508596.

9. Gérvas J. Prevención cuaternaria en ancianos. Rev Esp Geriatr Gerontol. 2012;47(6):266-269. http://dx.doi.org/10.1016/j.regg.2012.07.001. PMid:23062686.

10. Lenzer J, Brownlee S. US expert panel recommends against prostate cancer screening. BMJ. 2011;343:d6479. http://dx.doi.org/10.1136/bmj.d6479. PMid:21987717.

11. Brownlee S, Lenzer J. Can cancer ever be ignored? [Internet]. 2011 Oct 5 [última consulta el 2014 Oct 19]. The New York Times Magazine; 2011. Disponible en: www.nytimes.com/2011/10/09/magazine/can-cancer-ever-be-ignored.html?_r=2\&ref=magazine. 
12. Sullivan T. Evidence does not support blanket prostate cancer testing recommendation [Internet] 2013 Sept 5 [última consulta el 2014 Oct 19$].$ Healthy Debate; 2013.

Disponible en: http://healthydebate.ca/opinions/a-popular-belief-that-early-detection-of-cancer-is-best-does-not-make-it-so.

13. Moyer VA, U.S. Preventive Services Task Force. Screening for prostate cancer: U.S. Preventive Services Task Force recommendation statement. Ann Intern Med. 2012;157(2):120-134. http://dx.doi.org/10.7326/0003-4819-157-2-201207170-00459. PMid:22801674.

14. Welch HG, Black WC. Using autopsy series to estimate the disease "reservoir" for ductal carcinoma in situ of the breast: how much more breast cancer can we find? Ann Intern Med. 1997;127(11):1023-1028. http://dx.doi.org/10.7326/0003-4819-127-11-199712010-00014. PMid:9412284.

15. Borzekowski DL, Guan Y, Smith KC, Erby LH, Roter DL. The Angelina effect: immediate reach, grasp, and impact of going public. Genet Med. 2014;16(7):516-521. http://dx.doi.org/10.1038/gim.2013.181. PMid:24357847.

16. Arie S. Uruguay's mandatory breast cancer screening for working women aged 40-59 is challenged. BMJ. 2013;346:f1907. http://dx.doi.org/10.1136/bmj.f1907.

17. Pizzanelli Báez EM. Principios Éticos y Prevención Cuaternaria: ¿es posible no proteger el ejercicio del principio de autonomía? Rev Bras Med Fam Comunidade. 2014;9(31):169-73. http://dx.doi.org/10.5712/rbmfc9(31)852.

18. Welch HG. Should I be tested for cancer? Maybe not, and here's why. Berkeley: University of California Press; 2004.

19. Greaves M. Does everyone develop covert cancer? Nat Rev Cancer. 2014;14(4):209-210. http://dx.doi.org/10.1038/nrc3703. PMid:25688403.

20. Zahl PH, Gøtzsche PC, Mæhlen J. Natural history of breast cancers detected in the Swedish mammography screening programme: a cohort study. Lancet Oncol. 2011;12(12):1118-1124. http://dx.doi.org/10.1016/S1470-2045(11)70250-9. PMid:21996169.

21. Brodersen J, Schwartz LM, Woloshin S. Overdiagnosis: how cancer screening can turn indolent pathology into illness. APMIS. 2014;122(8):683689. http://dx.doi.org/10.1111/apm.12278. PMid:24862511.

22. Welch HG; Schwartz LM; Woloshin S. Are increasing 5-year survival rates evidence of success against cancer? JAMA. 2000;283:2975-2978. PMID: 10865276.

23. Esserman L, Shieh Y, Thompson I. Rethinking screening for breast cancer and prostate cancer. JAMA. 2009;302(15):1685-1692. http://dx.doi.org/10.1001/jama.2009.1498. PMid:19843904.

24. Sandblom G, Varenhorst E, Rosell J, Löfman O, Carlsson P. Randomised prostate cancer screening trial: 20 year follow-up. BMJ. 2011;(31):342:d1539. http://dx.doi.org/10.1136/bmj.d1539.

25. Hewitson P, Glasziou P, Irwig L, Towler B, Watson E. Screening for colorectal cancer using the faecal occult blood test, Hemoccult. Rev. Cochrane Database Syst. 2007;(1):CD001216. PMID: 17253456

26. Kronborg O, Fenger C, Olsen J, Jørgensen OD, Søndergaard O. Randomised study of screening for colorectal cancer with faecal-occult-blood test. Lancet. 1996;348:1467-1471. PMID: 8942774.

27. Brenner H, Tao S, Haug U. Low-dose aspirin use and performance of immunochemical fecal occult blood tests. JAMA. 2010;304(22):2513-2520. http://dx.doi.org/10.1001/jama.2010.1773. PMid:21139112.

28. Sacket D. Hormone replacement therapy. The arrogance of preventive medicine. CMAJ. [Internet]. 2002 [última consulta el 2015 Jun 17];167(4):363-364. Disponible en: http://www.cmaj.ca/content/167/4/363.full.pdf.

29. Menéndez E. El modelo médico y la salud de los trabajadores. Salud Colectiva. 2005;1(1):9-32.

30. Halfmann D. Recognizing medicalization and demedicalization: discourses, practices, and identities. Health (London). 2012;16(2):186-207. http://dx.doi.org/10.1177/1363459311403947. PMid:21540251. 